\title{
Review Paper: Emerging vertical-alignment liquid-crystal technology associated with surface modification using UV-curable monomer
}

\author{
Seung Hee Lee (SID Fellow) \\ Sung Min Kim (SID Member) \\ Shin-Tson Wu (SID Fellow)
}

\begin{abstract}
The recent development of polymer-induced pretilt angle in multi-domain vertical-alignment liquid-crystal (LC) structures is reviewed. To create a small but well-defined pretilt angle, $\sim 0.1 \mathrm{wt} . \%$ of a photo-curable monomer was mixed in an LC host and a bias voltage was applied to reorient the LC directors within each domain. The monomers are polymerized near the substrate surfaces by UV exposure. The formed polymer layers change the surface pretilt angle of the LC from $90^{\circ}$ to about $89^{\circ}$ with a defined azimuthal orientation. Consequently, within each domain the LC reorientation direction responding to the external field is well-defined which leads to faster rise time and higher transmittance. This new technology overcomes the long standing problems of conventional MVA devices and is therefore expected to play a dominant role in the future.
\end{abstract}

Keywords - Multi-domain vertical alignment, liquid crystal, polymerization, surface tilt angle.

DOI \# 10.1889/JSID17.7.551

\section{Introduction}

At present, two major wide-view liquid-crystal (LC) approaches are commonly practiced: homogenous alignment (HA) and vertical alignment (VA) in the initial state. The HA camp includes in-plane switching (IPS) $)^{1,2}$ and fringe-field switching (FFS),, 3 in which the LC directors essentially rotate in plane so that wide view is a built-in feature. While in the VA camp, multi-domain VA (MVA) using protrusion ${ }^{5-8}$ and patterned VA $(\mathrm{PVA})^{9,10}$ are the two major approaches.

In both types of VA devices, the LCs are vertically aligned with respect to the substrate with the help of a polymer alignment layer under crossed polarizers and their normalized transmittance $T / T_{o}$ can be described as

$$
T / T_{o}=\sin ^{2} 2 \psi(V) \sin ^{2}\left[\pi d \Delta n_{\text {eff }}(V) / \lambda\right],
$$

where $\psi$ is the angle between one of the transmission axes of the crossed polarizers and the LC director, $\Delta n_{\text {eff }}$ is the voltage-dependent effective birefringence of the LC medium, and $\lambda$ and $T_{o}$ are the wavelength and intensity of the incident light, respectively. In the absence of an electric field, $\psi$ $=0^{\circ}$ and the device appears black. In the presence of an electric field, the effective retardation $\left(d \Delta n_{\text {eff }}\right)$ starts to occur. Ideally, the transmittance will reach a maximum at $\psi=45^{\circ}$

In both MVA and PVA devices, the LC directors are reoriented by the oblique fields. In MVA, the oblique field is generated by a protrusion and patterned pixel electrode, whereas in PVA the oblique field originates from a patterned common and pixel electrode, as depicted in Fig. 1. Both MVA and PVA devices have been successfully com-

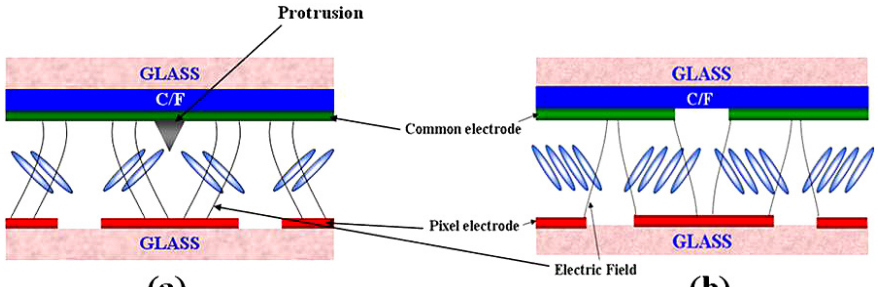

(a)

(b)

FIGURE 1 - Cross-sectional view of (a) MVA and (b) PVA devices.

mercialized; however, their performance and cost still need to be improved.

Recently, a new type of VA technology which enables the control of the surface pretilt angle of the LC by using a UV curable monomer was introduced. This technique improves the rise time and transmittance and has become a strong contender for future wide-view VA technology.

\section{Major issues in VA technologies}

Let us first describe the general problems associated with VA devices and the demerits of conventional MVA and PVA devices.

In the initial state, the LC directors are perfectly aligned vertically in the PVA device because the surfaces of the top and bottom substrates are almost flat. Thus, it shows a completely dark state because $\psi=0^{\circ}$. However, in the MVA device, there is a small amount of effective retardation in the LC layer with $\psi \neq 0$, due to the existence of protrusions. Then, light leakage occurs in the dark state near the protrusions, thereby decreasing the contrast ratio in the normal direction, which is an intrinsic problem.

S-H. Lee and S-M. Kim are with the Polymer Fusion Research Center, Department of Polymer Nano-Science and Technology, Chonbuk National University, Deokjinku, Deokjindong 1ga 664-14, Chonju, Chonbuk 561-756, Korea; telephone +82-63-270-2343, fax -2341, e-mail: 1sh1@chonbuk.ac.kr. $\mathrm{S}$-T. Wu is with the College of Optics and Photonics, University of Central Florida, Orlando, FL, USA.

(c) Copyright 2009 Society for Information Display 1071-0922/09/1707-0551\$1.00 
Further, the relatively strong light leakage occurs in the dark state in the oblique viewing direction, but this can be minimized by using proper optical compensation films between the LC layer and polarizer. ${ }^{11,12}$

The next problem is associated with the LC reorientation when the voltage exceeds a threshold. The vertically aligned LC tilts downward in the direction perpendicular to oblique electric fields with the aplication of a bias voltage, starting near the protrusions and patterened electrodes, and this deformation extends over the entire pixel area as time passes. In order to improve the rise time, overdriving technology through dynamic capacitance compensation (DCC) ${ }^{13}$ and DCC $\mathrm{II}^{14}$ was proposed; however, this approach was not satisfactory. The intrinsic problem associated with the slow rise time comes from the perfect vertical alignment of the LC except near the protrusions, so that the LC can reorient in any azimuthal directions when a vertical field is applied, as described in Fig. 2(a). Therefore, if the surface alignment layer is modified to have a given pretilt angle, then the LC directors will always have a predetermined reorientation direction so that the rise time will be improved, as Fig. 2(b) shows.

Another issue in the LC reorientation of a MVA cell is that the LC needs to tilt downward in at least four directions in order to show wide-view characteristics and the tilting direction should be $\psi=45^{\circ}$ to maximize the transmittance. Therefore, the precise control of the reorienting direction according to the applied voltage is necessary. However, with the present approaches used in MVA and PVA devices, the reorienting direction of the LC cannot be controlled perfectly so as to maximize the electro-optic performances of the display because the LC is vertically aligned and is controlled only by the protrusion or patterned electrodes.

In addition, forming a protrusion or patterned electrode on the top substrate is an extra process compared to the conventional manufacturing process for twisted nematics, which increases the cost. Moreover, the device assembly requires high accuracy registration in terms of the margin between the top and bottom substrates in order to control the LC perfectly, which makes it difficult to manufacture.

Consequently, the development of new VA technology which shows high performance with low cost and easy manufacturing is desired. This was recently achieved by using a UV-curable monomer. The LCDs that adopt this technology are now starting to appear.

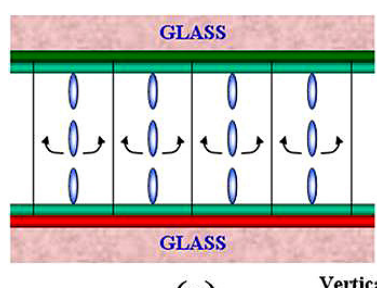

(a)

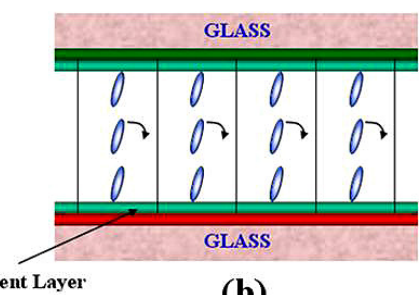

(b)
FIGURE 2 - Schematic drawings showing LC reorientation in response to a vertical electric field: (a) perfect vertical alignment and (b) vertical alignment with a small pretilt angle.

\section{$3 \quad$ New wide-view VA technology using UV-curable monomer}

\subsection{History of related technology}

Research into composites of LCs and polymers for use as gas-permeable membranes and electrically controllable displays began in the late 1970s. Especially, the latter, known as polymer-dispersed liquid crystal (PDLC) with a polymer content of over $10 \%$ were extensively studied in the 1980s, and even now are being studied and commercialized for switchable windows. ${ }^{15}$ Since then, several works on the polymer stabilization of LC orientation for twisted-nematic (TN) and ferroelectric LCDs with a monomer content of 2 wt.\% were reported in the 1990 s. ${ }^{16-18}$ Later on, many attempts to stabilize the LC director using polymer networks ${ }^{19}$ were reported, but the concept of polymer stabilization was mainly used to stabilize the surface and bulk of the LC layer through polymer networks. In transmissive LCDs, high contrast ratio is one of the key requirements for high-performance displays. However, the technology that has been introduced has polymer networks in the bulk of the LC layer and, thus, there is a mismatch in the refractive index between the LC and polymer. This causes light scattering in the dark state which degrades the contrast ratio. Therefore, minimizing the polymer content in the LC host mixture is very important to maintain the high image quality of the LCDs, while stabilizing the LC directors as needed.

The first work on polymer stabilization in VA mode appeared in 1998. ${ }^{20,21}$ Especially, Kume et al. ${ }^{20}$ reported for the first time that the alignment direction and surface tilt angle can be controlled by using a polymer, as demonstrated by their SEM photograph of the alignment layer and model of the polymer-controlled surface tilt angle. In both of their reports, they used a position controllable resin spacer and gibbous lattice structure. Then, in 2004, Hanaoka et al. ${ }^{22}$ reported polymer-sustained alignment (PSA) technology, in which multiple LC directors in the voltage-on state are achieved using a minutely patterned electrode with a fishbone shape on the pixel electrode, and the tilt angle of the LC on the VA layer was stabilized by using a photo-curable monomer. They claimed that the proposed device exhibits a higher transmittance, better dark state, and faster response time as compared to the MVA device. In 2007 and 2008, our group $^{23-26}$ reported the surface polymer stabilization of the LC director in a PVA device and other VA devices using a reactive mesogen (RM), showing a considerable improvement in the rise time, in which the doping amount of the RM was about $0.1 \mathrm{wt} . \%$ in the LC host. Recently, Hsu et al. ${ }^{27}$ reported detailed results on the optical performance of a polymer-stabilized mobile MVA-LCD according to the curing process of the reactive monomer with a doping amount of 0.05 wt. $\%$. 


\subsection{Key technologies in polymer-stabilized VA LCDs}

\subsubsection{Cell design for triggering the formation of multi-domain LC directors}

In order to stabilize or fix the LC director in the desired direction on the VA layer, the trigger, which forms the multiple domains of the LC directors when a voltage is applied, must exist. This trigger should be fabricated as easily as possible without an additional process and should not deteriorate the level of the dark state. Summarizing the reported works on VA technologies associated with polymer stabilization depending on triggers, the approaches can be divided into three categories: (1) polymer wall or gibbous lattice structures, (2) no patterned common electrode on the top substrate and a minutely patterned fishbone-shaped pixel electrode on the bottom substrate, and (3) patterned electrodes on both substrates.

\subsubsection{Control of surface pretilt angle}

The polymer-stabilized technique modifies the surface of the VA layer which, in turn, changes the pretilt angle from $90^{\circ}$ to less than $90^{\circ}$. The resultant surface pretilt change should be as small as possible; otherwise the $d \Delta n_{\text {eff will be }}$ affected in the normal direction, causing the light leakage which degrades the device contrast ratio. In addition, a small pretilt angle should be generated so that $\psi$ is equal to $45^{\circ}$ in the voltage-on state in order to obtain maximum transmittance. According to previous reports, ${ }^{23,27}$ the surface pretilt angle is dependent on the bias voltage as well as the UV curing dosage.

\subsubsection{Minimizing UV curing time and residual monomers}

In the manufacturing process, the curing time should be minimized to maximize the productivity of mass production. However, this seems relatively difficult to achieve because the curing condition plays a very important role in controlling the surface pretilt angle. A fast curing may leave some of the monomer uncured, which could be a source for the undesirable image sticking.

\subsection{Review of previous works based on published results}

\subsubsection{Polymer-stabilized VA technology with polymer wall}

Figure 3 shows the cell structure of a MVA device in which a position-controlled square-shaped resin spacer exists between the top and bottom substrates, playing the role of triggering and providing cell-gap control. The LC with a negative dielectric anisotropy $(\Delta \varepsilon<0)$ is filled, a vertical electric field

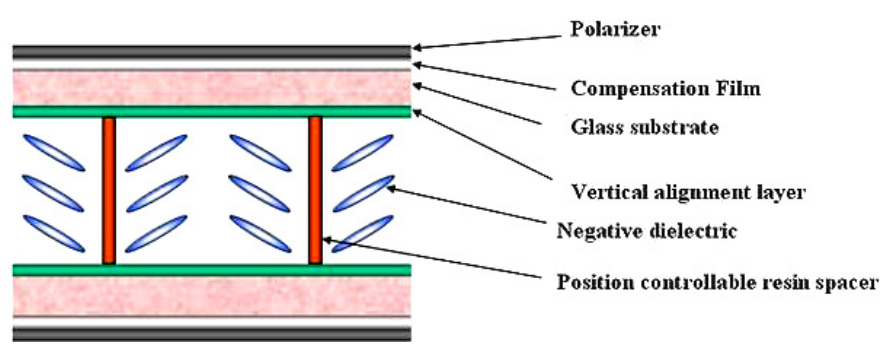

FIGURE 3 - Cell structure of N-type ASM mode panel (Ref. 20).

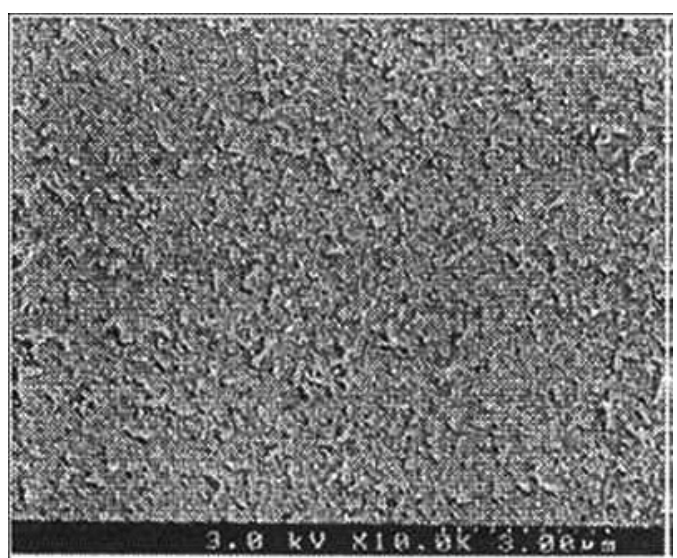

FIGURE 4 - SEM photograph of alignment layer showing grain-shaped substances (Ref. 20).

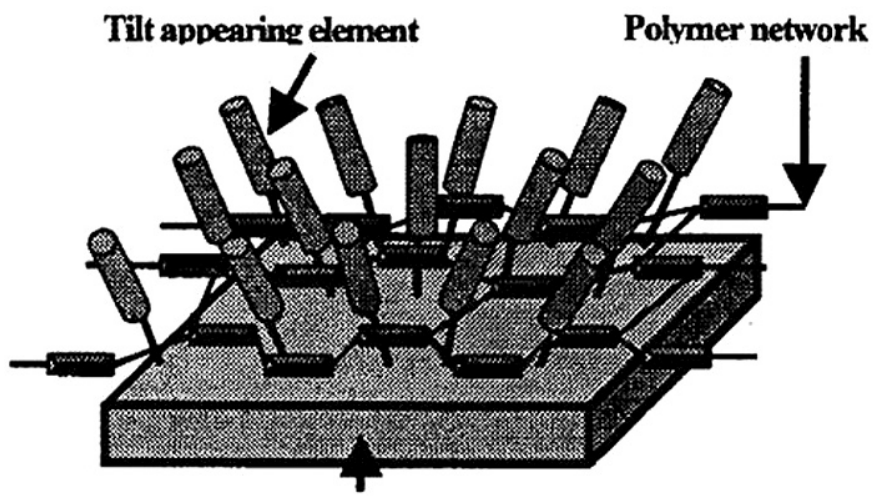

(a) Vertical alignment layer

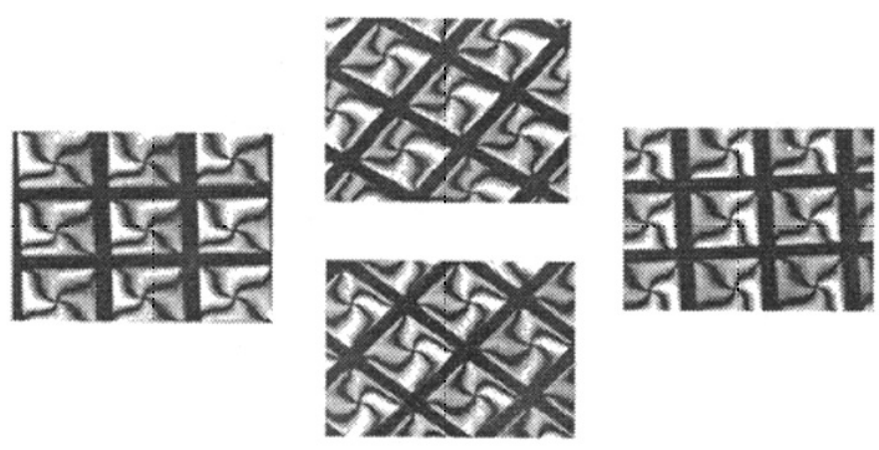

(b)

FIGURE 5 - (a) Model of polymer-controlled tilt-appearing element with a polymer network on the surface of a VA layer and (b) four brushed-schlieren LC texture showing multiple domains (Ref. 20). 


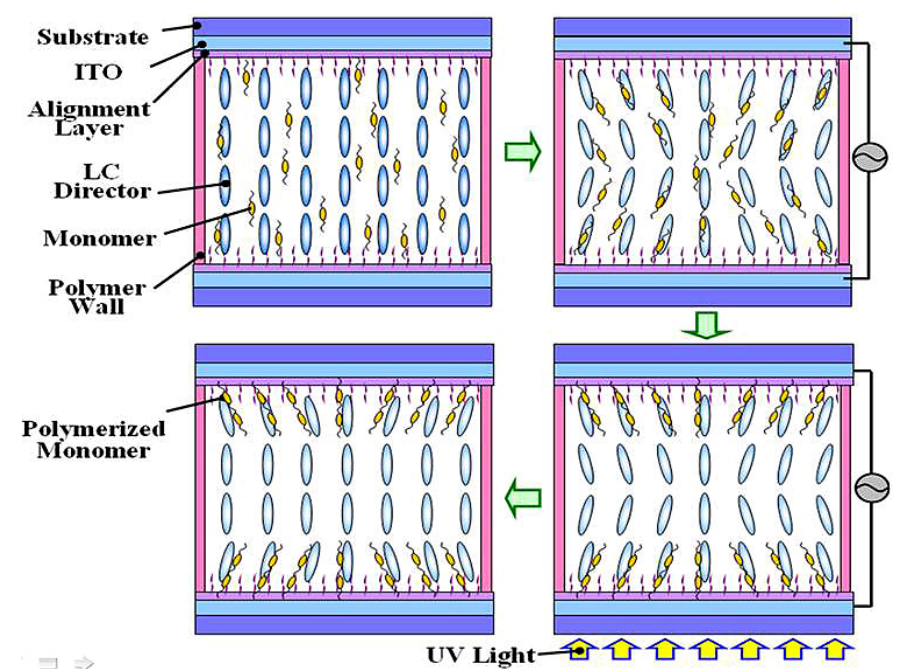

FIGURE 6 - Schematic diagram of surface polymer stabilization in the VA cell in order to achieve defined pretilt angles on the VA layer (Ref. 25).

is applied as in a TN device, and a photo-curable monomer is doped in the LC. ${ }^{18}$ This device was referred to as the $\mathrm{N}$-type ASM mode. In this device, once the operating voltage is applied, the LC directors will be axially symmetrically aligned due to the polymer wall. However, the time-resolved LC texture might show a change in LC texture with time, although this issue was not clarified in Ref. 18. Hence, the device shows a wide viewing angle, but the rise time is slow. The authors explained that after setting the LC in the desired direction and at the desired tilt angle, polymerization was carried out by UV exposure and the existence of grainshaped substances was confirmed on the VA layer, as shown in Fig. 4. After this process, the authors predicted that the alignment direction and tilt angle of the LC would be fixed by the polymer, as shown in Fig. 5(a), leading to a stable multi-domain LC texture [see Fig. 5(b)].

More detailed information is given in Refs. 25 and 26 for similar cell structures where a chiral-doped LC was mixed with 0.1 wt.\% of the RM, the LC was surrounded by a polymer wall made of photoresist, and a vertical field was applied to the cell. The monomer content was minimal, in order to avoid any light scattering from the polymerized mesogen and to have the polymer network only on the surface of the VA layer. As described in Fig. 6, the RM was vertically aligned to be parallel to the LC directors in the

(a)

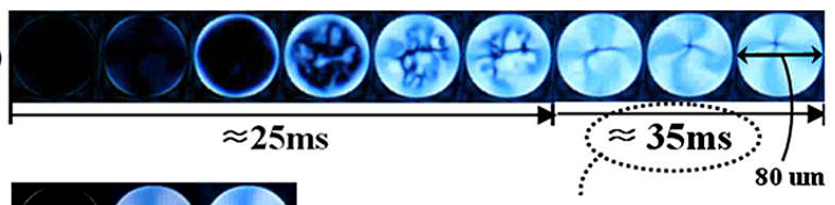

(b)
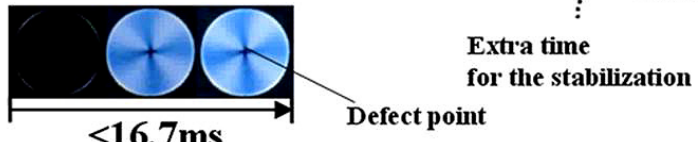

FIGURE 7 - Time-resolved LC textures needed to reach $90 \%$ of maximum transmittance in the VA cell without (a) and with (b) polymer stabilization (Ref. 25).

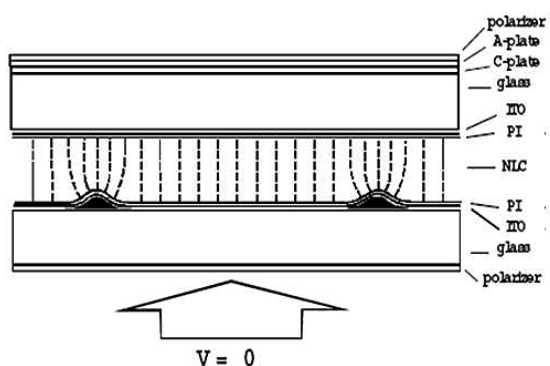

(a)

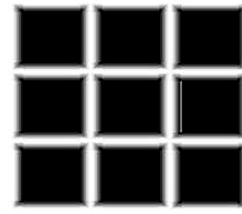

(b)
FIGURE 8 - (a) Cell structure of polymer-stabilized VA cell and (b) top view of the gibbous lattice structure (Ref. 21).

initial state, and then a voltage is applied to reorient the LC directors and finally a multi-domain configuration is achieved due to the polymer wall. When the LC directors reach the stable state for maximum light efficiency, UV is irradiated to let the RM migrate to the surface of the VA layer and, subsequently, the LC is polymerized at the surface, forming the surface tilt angle. As a result, even after the applied voltage is released, the surface tilt angle is fixed by the additional polymer layer.

The time-resolved LC textures without and with polymer stabilization of the LC cells when a voltage is applied to obtain the white state are shown in Fig. 7. In the VA cell without polymer stabilization, the LC tilts down with a twist angle of $90^{\circ}$ from top to bottom because $d / p=0.25$ ( $p$ is the LC pitch length); however, the azimuthal tilting-down direction of the LC is not defined, so that the LC molecules collide with each other, showing an undefined texture before reaching the stabilized texture. The LC cell with $80-\mu \mathrm{m}$ polymer walls takes approximately $25 \mathrm{msec}$ for the fourbrush texture to appear and another $35 \mathrm{msec}$ for it to be stabilized. However, in the VA cell with polymer stabilization, the defect point is fixed at the center of the pixels, indicating that there are no collisions between the molecules. As a result, the entire process takes less than $17 \mathrm{msec}$, owing to the defined tilting direction.

Instead of using a polymer wall, another approach for triggering the deformation of the LC directors is to use a type of protrusion, called a gibbous lattice, as Fig. 8 shows. In this cell, in which a protrusion with a square-shaped lattice exists, a vertical field is used and the LC is mixed with diacrylate monomer $(<5$ wt.\%). In this case, once a high voltage is applied, the LC directors do not know where to tilt down, so an irregular LC texture appears. But with polymer stabilization, a regular texture appears, giving rise to a shortened rise time, as shown in Fig. 9.

\subsubsection{Polymer-stabilized VA technology without a polymer wall or protrusion}

Forming a protrusion or polymer wall requires an additional process compared to the fabrication of a $\mathrm{TN}$ device, and the non-perfect vertical alignment of the LC directors near the protrusion or walls causes light leakage in the dark state. A 


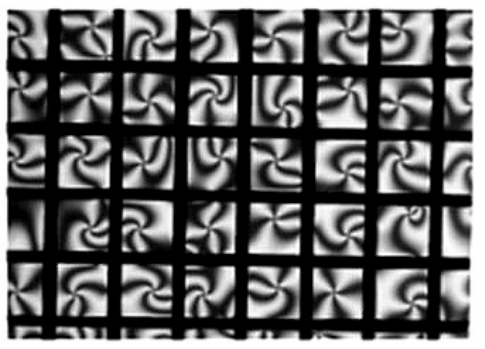

(a)

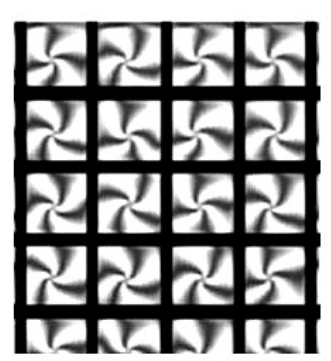

(b)
FIGURE 9 - Optical texture of the cell without (a) and with (b) polymer stabilization (Ref. 21).

new type of MVA refered to as a PSA-LCD was proposed in 2004 with the cell structure shown in Fig. $10 .^{22}$

In this device, the common electrode is not patterned; instead, the pixel electrode is patterned minutely in the form of slits, and an appropriate concentration of UV-curable monomer is added to the negative $\Delta \varepsilon \mathrm{LC}$ host. As in the previous cases, the polymer layer is formed above the surface of the VA layer by UV exposure. Once the stable reorientation of the LC is obtained by applying a voltage, the fixed tilt angle of the polymer layer makes LC reorientation easy when the voltage is applied.

One of the key issues in this device is how stable LC orientation can be achieved with $\psi \sim 0^{\circ}$ in the dark state and $\psi=45^{\circ}$ in the bright state. In the conventional MVA and PVA devices, where the width of the slit is smaller than that of the pixel electrode, the LC directors are reoriented perpendicular to the slit direction. However, in the PSA-LCD with its minutely patterned ITO slits, the LC directors are reoriented parallel to the slit direction, as schematically drawn in Fig. 10(b). Hanaoka et al. proposed a pixel structure for a 17-in. PSA-LCD with patterned electrodes having a fishbone shape, as shown in Fig. 11.

In order to understand how the LC reorientation evolves according to time when an appropriate voltage is applied, a 3-D simulation was performed using a simulator (Sanayi System, Korea). Immediately after the voltage is applied, the LC directors try to reorient perpendicular to the slit direction [see Fig. 12(b)], and as time evolves, the LCs collide with each other, due to the small width of the pixel electrodes, and the LC starts to tilt down in the slit direction [see Fig. 12(c)]. Finally, after a sufficient relaxa-

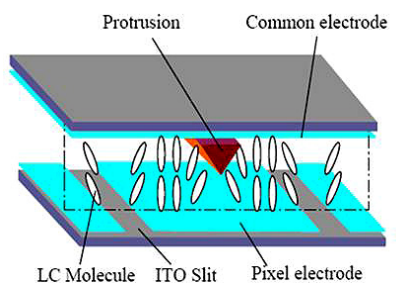

(a)

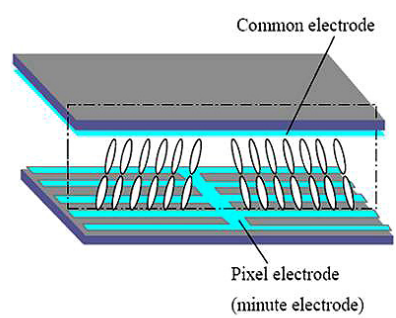

(b)
FIGURE 10 - Pixel structure of (a) conventional MVA-LCD and (b) PSA-LCD with minutely patterned ITO slits (Ref. 22).

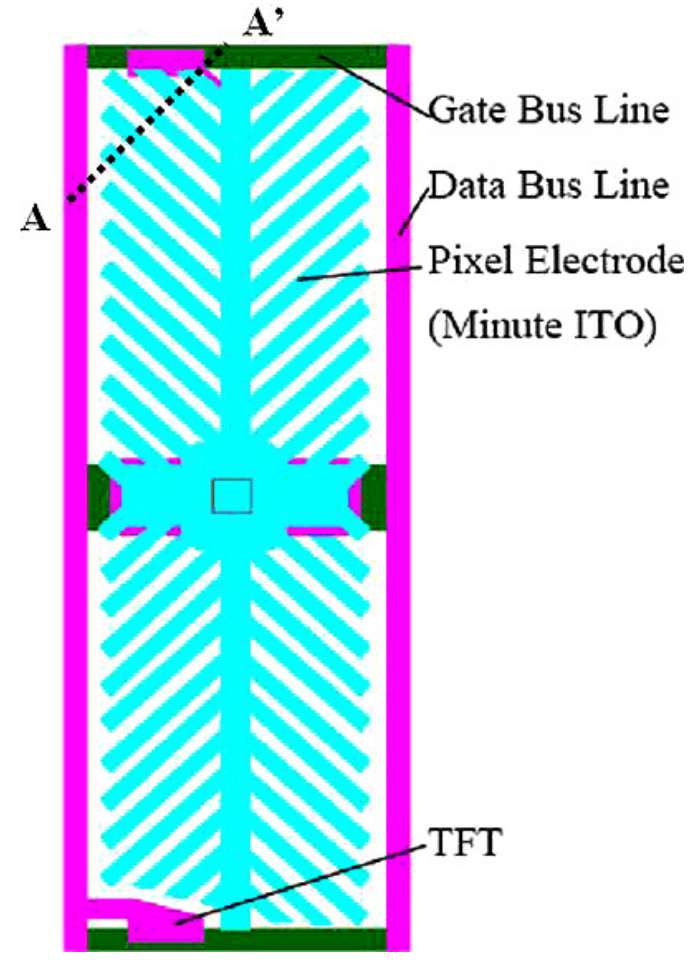

FIGURE 11 - Pixel structure of PSA-LCD (Ref.22).

tion time, the LC is reoriented toward the slit direction, as schematically described in Fig. 12(d). Figure 13 shows the top view of one pixel, demonstrating the transmittance distribution when the operating voltage is applied. In the beginning, the LC directors collide with each other, generating a large amount of disclination lines. However, after a sufficient amount of time has passed, they become stabilized so that a uniform distribution of the transmittance is achieved. Once the director is stabilized, UV is irradiated while the voltage is applied, and then the monomer is polymerized at the surface of the VA layer, causing the direction and pretilt angle of the LC to be fixed. They reported that the PSA-LCD shows a $25 \%$ improvement in brightness and $40 \%$ improvement in contrast ratio, owing to the elimination of the light

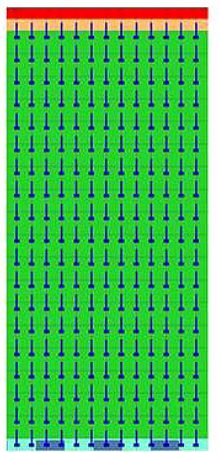

(a)

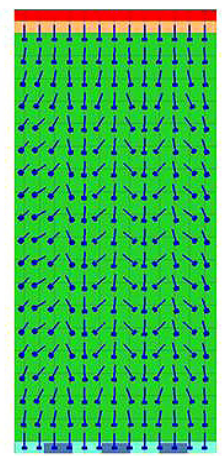

(b)

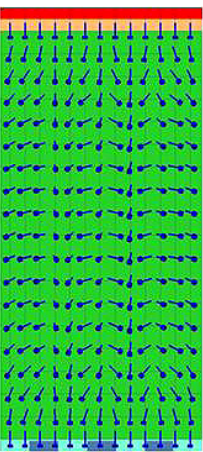

(c)

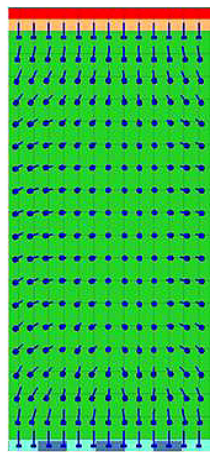

(d)
FIGURE 12 - Simulated results of time-resolved LC reorientation when a certain voltage is applied to the PSA-LCD along direction A-A': (a) $t=$ $0 \mathrm{msec}$, (b) $t=10 \mathrm{msec}$, (c) $t=20 \mathrm{msec}$, and (d) $t=2 \mathrm{sec}$. 


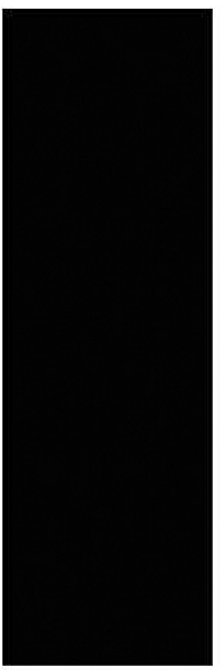

(a)

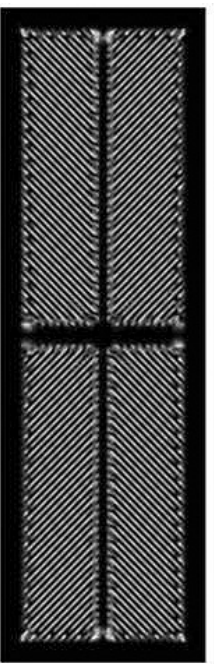

(b)

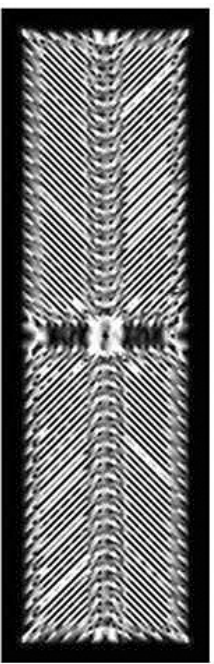

(c)

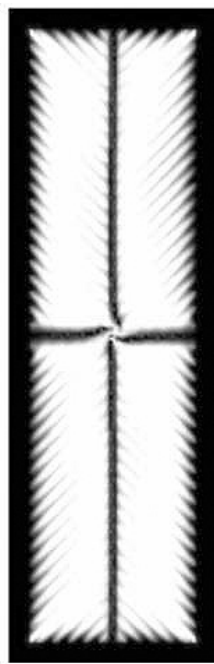

(d)
FIGURE 13 - Simulated results of time-resolved texture when a certain voltage is applied to the PSA-LCD along direction $A-A^{\prime}$ : (a) $t=0$ msec, (b) $t=10 \mathrm{msec}$, (c) $t=20 \mathrm{msec}$, and (d) $t=2 \mathrm{sec}$.

leakage from the protrusions, and also the response times in the gray scales are improved.

Another work 27 in which the polymer-stabilizing technique is applied to mobile LCDs was reported. The concept of the device seems to be similar to that of the PSA-LCD, although the detailed pixel structure was not revealed. The device uses a very low concentration $(\sim 0.05 \mathrm{wt} . \%)$ of monomer in the LC host. Relatively detailed information was presented on how the electro-optical performance depends on the curing conditions, such as voltage, UV-dosage, and the type of monomers used. In addition, the authors emphasized the importance of the UV curing process and dosage, and explained the two-step UV curing process; the first step serves to fix the tilt angle of the LC by the reactive monomer and the second step is to remove the residual monomer. They also commented that the selection of a proper monomer plays an important role because it determines the alignment anchoring force, and a stronger anchoring force on the

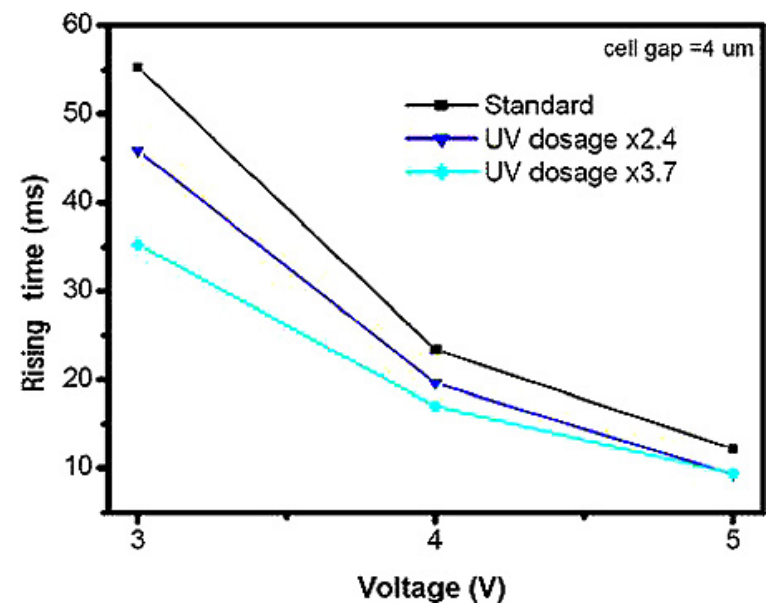

FIGURE 14 - UV dosage vs. rise time (Ref. 27).

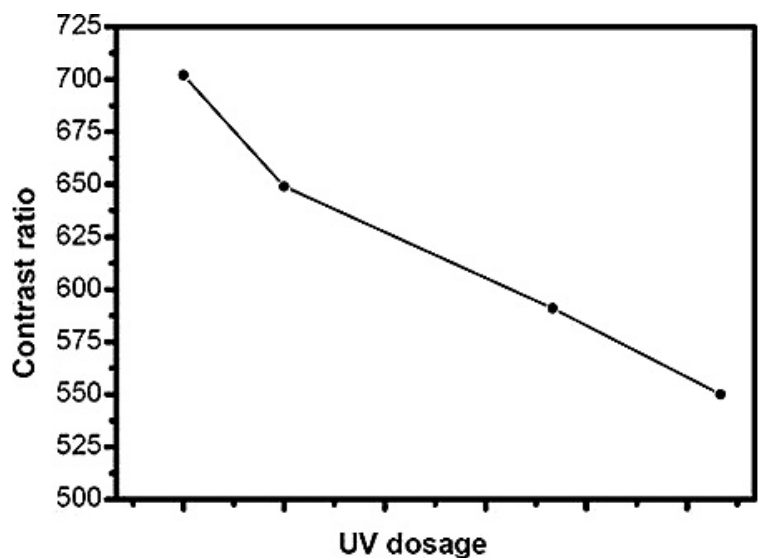

FIGURE 15 - Relationship between contrast ratio and UV dosage (Ref. 27).

monomer/polymer layer allows a similar level of performance to be achieved at a lower UV dosage.

Figure 14 shows the relationship between the UV dosage and rise time. ${ }^{27}$ As indicated, the rise time decreases with increasing UV exposure time, indicating that a larger UV dosage induces a better molecular alignment. A large UV dosage increases the surface tilt angle, so that the rise time is shortened, but the contrast ratio will decrease with increasing UV dosage, due to the effective $\psi$ and $d \Delta n_{\text {eff }}$ in the normal direction, as presented in Fig. 15. Unfortunately, the response time and contrast ratio have a trade-off relationship. The curing voltage is also a very important factor to determine the surface tilt angle because a higher percentage of the LC can be finely controlled with a higher applied voltage. Nevertheless, when the curing voltage was varied from 15 to $45 \mathrm{~V}_{\mathrm{rms}}$, only a slight difference during full switching from black to white was observed by the authors, but an improvement in all of the gray-level response times was observed, as shown in Fig. 16. One noticeable point is that the voltage here does not appear to be the real voltage applied to the LC because the operating voltage of the device cannot be so high.

The authors also evaluated the alignment ability of the polymer layer by employing a new material, AU004. ${ }^{27}$ With

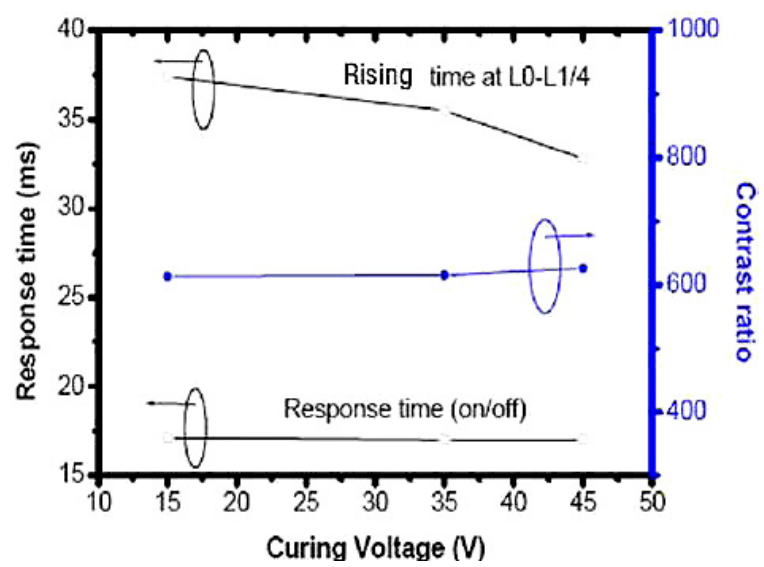

FIGURE 16 - Response time and contrast ratio according to the curing voltage (Ref. 27). 


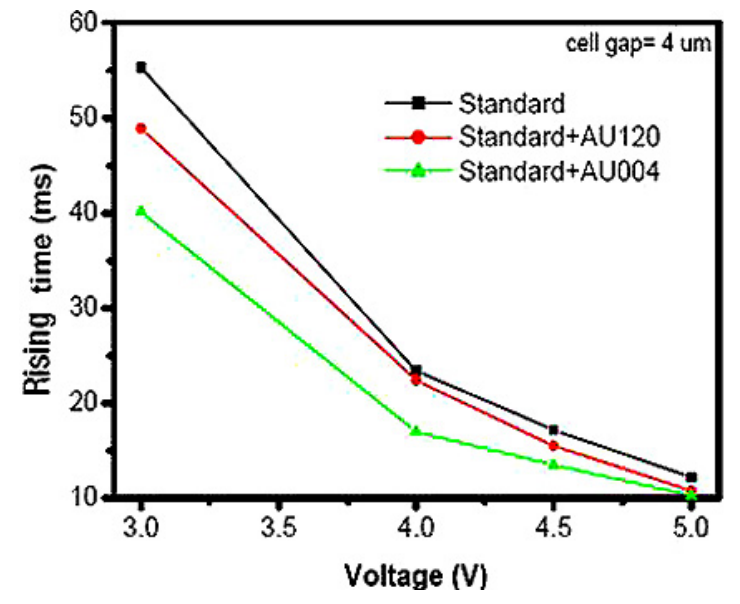

FIGURE 17 - Improvement of rise time with new materials (Ref. 27).

this new material, the interaction between the LC and polymer was found to be stronger, which indicates that the LC molecules can achieve the pretilt angle more efficiently. Hence, a larger improvement corresponding to a $\sim 30 \%$ reduction in the rise time was achieved, as shown in Fig. 17.

The last case of the application of the polymer-stabilizing technique to VA mode was associated with the PVA device. ${ }^{23,24,28}$ In this device, the common and pixel electrodes are patterned alternatively, as shown in Fig. 1, and the RM is doped into the LC. The LC reorientation is strongly dependent on the applied voltage in a normal PVA cell because here the triggering required for deforming the LC is only the oblique field generated by the patterned electrodes. Therefore, in this device, both curing voltage and monomer content with curing dosage play a very important role to determine the surface tilt angle. For the comparison purpose, $5 \mathrm{~V}$ was applied in each case and the time-resolved LC textures were observed, as shown in Fig. 18. When the curing voltage is low, $2.3 \mathrm{~V}$, with $0.1 \mathrm{wt} . \%$ of $\mathrm{RM}$, the pretilt

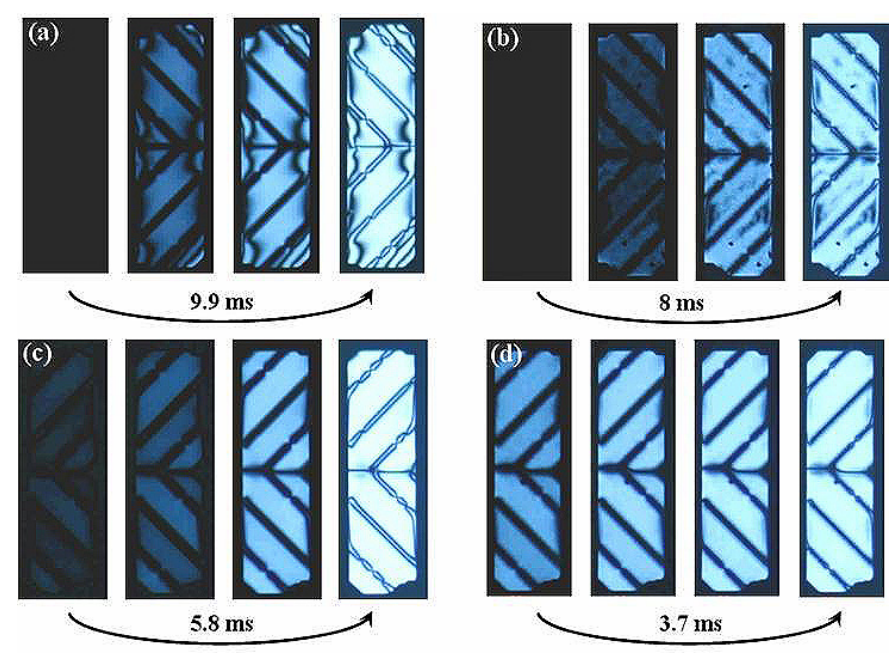

FIGURE 18 - Time-resolved LC textures with an applied voltage of 5 $\mathrm{V}$ : the surface-modified PVA cell cured at a specific voltage with different wt.\% of RM monomer: (a) $2.3 \mathrm{~V}, 0.1 \%$, (b) $2.3 \mathrm{~V}, 1 \%$, (c) $5.3 \mathrm{~V}, 0.1 \%$, (d) $5.3 \mathrm{~V}, 1 \%$ (Ref. 28). angle is formed by the polymer layer, but its direction does not correspond to $\psi=45^{\circ}$, as can be seen from the irregular dark lines [see Fig. 18(a)], which lowers the transmittance, and the rise time is rather slow, $9.9 \mathrm{msec}$, compared to the other cases. When the amount of RM is increased to $1 \mathrm{wt} . \%$, the LC texture evolves rather coarsely with not much improvement in the response time [see Fig. 18(b)]. Now, when the curing voltage is as high as $5.3 \mathrm{~V}$, the two cells with 0.1 and 1 wt.\% of RM show a considerable improvement in their rise time with a neat time-resolved texture. This is due to the high tilt angle formed by the polymer layer; however, this deteriorates the level of the dark state, such that strong light leakage is observed at 0 msec [see Figs. 18(c) and $18(\mathrm{~d})]$ and, thus, a severe decrease in contrast ratio is observed. The authors also claimed that the initial UV-curing intensity plays a very important role, although the total dosage is the same, and Fig. 19 shows an example of this. Although the RM content was very low, $0.1 \mathrm{wt} . \%$, using a strong UV intensity induces a fast polymerization of the RM and, hence, noticeable polymer chains along the direction perpendicular to the slit direction are observed, as shown in Figs. 19(a) and 19(b). The polymer chains formed in this way generate a very large pretilt angle of the LC and, therefore, strong light leakage is observed in the dark state, which is undesirable. For curing using a weak UV intensity, only grain-shaped polymer appears, but this is not noticeable under an optical polarizing microscope, as shown in Fig. 19(c).

Unlike the previous case, ${ }^{27}$ the surface tilt is strongly dependent on the UV-curing voltage in the PVA cell and the initial UV-curing intensity is also an important factor to control. Under optimized UV curing conditions such as the curing voltage, curing intensity, and monomer content, the PVA cell with surface polymer stabilization shows a considerable improvement not only in full switching from black to white, but also in all gray-level switching, as shown in Fig. 20. An improvement in rise time simply indicates that the surface tilt angle and direction of the LC over the entire surface area are defined so that the LC can reorient much more easily than that without a defined tilt angle when a voltage is applied.
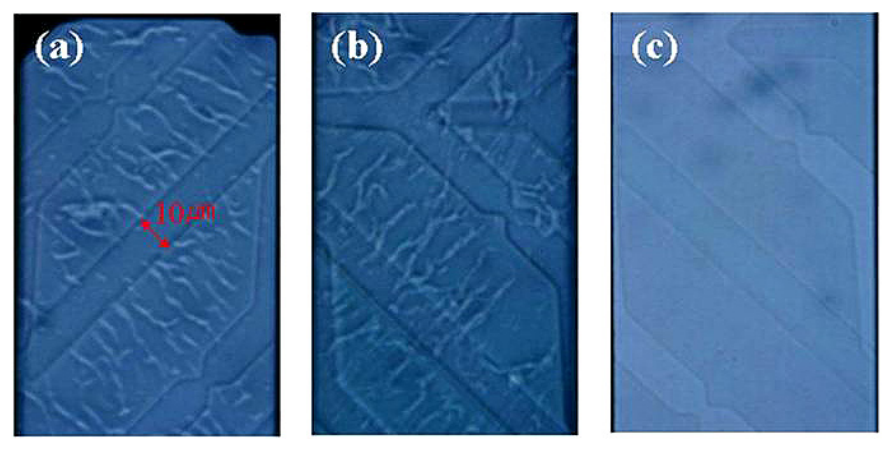

FIGURE 19 - Microphotographs of the inside surface of the PVA cell prepared at different UV-curing intensities: (a) $246.3 \mathrm{~mW} / \mathrm{cm}^{2} \times 30 \mathrm{~min}$, (b) $98.5 \mathrm{~mW} / \mathrm{cm}^{2} \times 74 \mathrm{~min}$, and (c) $5.0 \mathrm{~mW} / \mathrm{cm}^{2} \times 24$ hours (Ref. 28). 


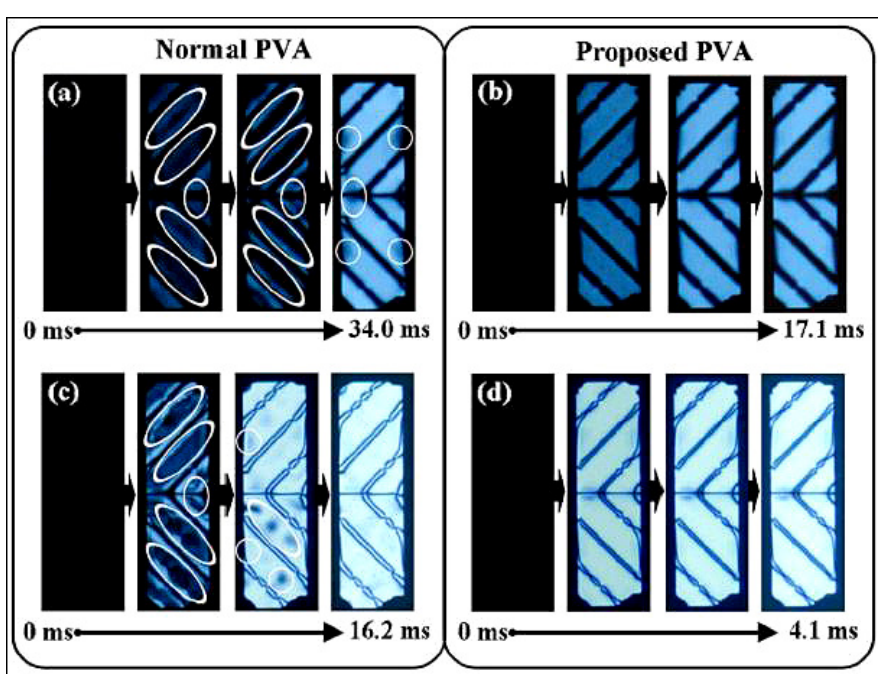

FIGURE 20 - Time-resolved LC textures in normal and polymerstabilized PVA cells: (a) and (b) when a mid-gray voltage is applied; (c) and (d) when a white voltage is applied; textures inside circles or ellipses indicate disclination lines due to collisions between LC (Ref.23).

\section{Summary}

This paper reviews the advancement in novel VA technologies associated with surface polymer stabilization which improves the electro-optic characteristics of the devices compared to conventional VA devices, especially in rise time. Unlike conventional polymer/LC composites which form polymer networks over the entire LC layer, in this technology the polymer layer exists only at the surface of the vertical-alignment layer in order to provide a defined pretilt angle and reorientation direction. The pretilt angle should be close to $90^{\circ}$ to minimize the retardation in the normal direction, in order to achieve a completely dark state and the direction should be $45^{\circ}$ with respect to the crossed polarizer to maximize the transmittance in the on-state. In order to achieve such a desirable polymer layer by the polymerization of the monomer, first the optimization of the pixel structure to form stable multiple LC directors through collisions between the LC molecules during switching needs to be achieved. Second, the UV-curing conditions such as the curing voltage, curing intensity, curing energy, and amount of monomer in the LC host need to be optimized. Finally, the development of monomers which can increase the anchoring force of the alignment layer is also important for achieving a faster response time.

The PSA-LCD not only exhibits improved electro-optic performance in comparison to conventional VA devices, but also eliminates the need to form protrusions in the MVA device and the patterning of the common electrode in the PVA device. Therefore, from the performance and cost viewpoints, the PSA-LCD device has advantages over other VA devices. We expect this new VA technology associated with surface polymer-stabilization will find widespread applications in the future.

\section{References}

1 M. Oh-e and K. Kondo, "Electro-optical characteristics and switching behavior of the in-plane switching mode," Appl. Phys. Lett. 67, 3895-3897 (1995)

2 J.-S. Y. et al., "Novel electrode structure in the super-IPS LC cell for high-aperture ratio," SID Symposium Digest 38, 752-755 (2007).

3 S. H. Lee et al., "Electro-optic characteristics and switching principle of a nematic liquid crystal cell controlled by fringe-field switching," Appl. Phys. Lett. 73, 2881-2883 (1998).

4 S. H. Lee et al., "A novel wide-viewing-angle technology: Ultra-trans view TM," SID Symposium Digest 30, 202-205 (1999).

5 K. Ohmuro et al., "Development of super-high-image-quality verticalalignment-mode LCD," SID Symposium Digest 28, 845-848 (1997).

6 C. W. Chen et al., "A LCD novel design for high contrast ratio," SID Symposium Digest 39, 133-135 (2008).

7 A. Takeda et al., "A super-high image quality multi-domain vertical alignment LCD by new rubbing-less technology," SID Symposium Digest 29, 1077-1080 (1998).

8 H. Seiberle and M. Schadt, "Photoalignment and photo-patterning of planar and homeotropic liquid-crystal-display configurations," J. Soc. Info. Display 8/1, 67-71 (2000).

9 K. H. Kim et al., "Domain divided vertical alignment mode with optimized fringe field effect," Proc. Asia Display, 383-387 (1998)

10 S. S. Kim et al., "An 82-in. ultra-definition 120-Hz LCD TV using new driving scheme and advanced super PVA technology," J. Soc. Info Display 17/2, 71-78 (2009).

11 J. Chen et al., "Optical simulation of electro-optical performance of low- $\Delta$ nd multi-domain TN displays," SID Symposium Digest 28 , 937-940 (1997)

12 D.-K. Yang and S.-T. Wu, Fundamentals of Liquid Crystal Devices (John Wiley \& Sons, Ltd., 2006).

13 K. H. Kim et al., IMID Digest, 58-61 (2001).

14 J. K. Song et al., "DCCII: Novel method for fast response time in PVA mode," SID Symposium Digest 35, 1344-1347 (2004).

15 P. S. Drzaic, Liquid Crystal Dispersions (World Scientific Publishing Co., 1995)

16 P. J. Bos et al., "A low-threshold-voltage polymer network TN device," SID Symposium Digest 24, 877 (1993).

17 Y. Iimura and S. Kobayashi, "Electro-optic characteristics of amorphous and super-multidomain TN-LCDs prepared by a non-rubbing," SID Symposium Digest 25, 915 (1994).

$18 \mathrm{H}$. Furue et al., "characteristics and driving scheme of polymer-stabilized monostable flcd exhibiting fast response time and high contrast ratio with gray-scale capability," SID Symposium Digest 29, 782-785 (1998).

19 S.-T. Wu and D.-K. Yang, Reflective Liquid Crystal Displays (John Wiley \& Sons, Ltd., 2001).

20 Y. Kume et al., "Advanced ASM mode (axially symmetric aligned microcell mode): Improvement of display performances by using negative dielectric liquid crystal," SID Symposium Digest 29, 1089-1092 (1998).

21 C.-K. Wei et al., "Wide-viewing-angle polymer stabilized homeotropically aligned (PSHA) LCD," SID Symposium Digest 29, 1080-1083 (1998)

22 K. Hanaoka et al., "A new MVA-LCD by polymer sustained alignment technology," SID Symposium Digest 35, 1200-1203 (2004).

23 S. G. Kim et al., "Stabilization of the liquid crystal director in the patterned vertical alignment mode through formation of pretilt angle by reactive mesogen," Appl. Phys. Lett. 90, 261910-261912 (2007).

24 E. J. Jeon et al., "Pretilt angle control for single cell gap transflective liquid crystal cells in vertical alignment mode," Proc. IDRC, 141-143 (2008).

25 S. G. Kim et al., "Trapping of defect point to improve response time via controlled azimuthal anchoring in a vertically aligned liquid crystal cell with polymer wall," J. Phys. D: Appl. Phys. 41, 055401-055404 (2008).

26 S. J. Hwang et al., "Surface polymer-stabilized vertically aligned liquid crystal cells with various polymer wall structures," Mol. Cryst. Liq. Cryst. 489, 237-245, (2008).

27 S.-F. Hsu et al., "Advanced-MVA mobile technology for fast-switching LCD displays," SID Symposium Digest 39, 503-506 (2008).

28 S. M. Kim et al., "Surface-modification on vertical alignment layer using UV-curable reactive mesogens," Jpn. J. Appl. Phys. 48, 032405-8, (2009) 


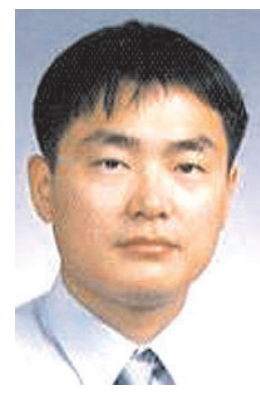

Seung Hee Lee received his B.S. degree in physics from Chonbuk National University in 1989 and Ph.D. degree from the Physics Department of Kent State University in 1994. In 1995, he joined the LCD division at Hyundai Electronics. Since then, he developed and commercialized new liquid-crystal devices, called "fringe-field switching (FFS)" mode. He was awarded "King of the Invention" twice in the company. In September of 2001, he became a professor of Chonbuk National University in Chonju, Korea. He was selected as a SID Fellow in 2008 and Best Research Professor of Chonbuk National University in 2007 and 2008.

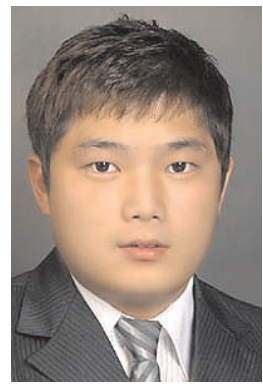

Sung Min Kim received his B.E. and M.E. degrees from the Department of Polymer Nano-Science and Technology from Chonbuk National University in 2007 and 2009, respectively. At present, he is taking his doctoral course in the same department. His main research field is the development of vertical-alignment liquid-crystal displays having high performance at low cost.

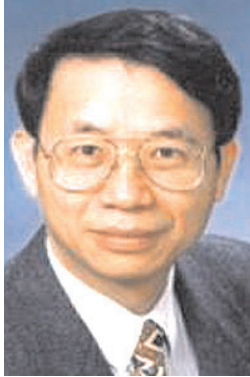

Shin-Tson $\mathbf{W u}$ is a PREP Professor at the College of Optics and Photonics, University of Central Florida. Prior to joining UCF in 2001, he worked at Hughes Research Laboratories (Malibu, California) for 18 years. He received his Ph.D. in physics from University of Southern California (Los Angeles, California, U.S.A.) and his B.S. in physics from National Taiwan University (Taipei, Taiwan). $\mathrm{He}$ is the recipient of 2008 SID Jan Rajchman prize and 2008 SPIE G. G. Stokes award. He is a Fellow of the IEEE, SID, OSA, and SPIE. He has co-authored 5 books: Introduction to Flat Panel Displays (Wiley, 2008), Fundamentals of Liquid Crystal Devices (Wiley, 2006), Introduction to Microdisplays (Wiley, 2006), Reflective Liquid Crystal Displays (Wiley, 2001), and Optics and Nonlinear Optics of Liquid Crystals (World Scientific, 1993), six book chapters, over 300 journal papers, and 60 issued patents. He was the founding Editor-In-Chief of the IEEE/OSA Journal of Display Technology. 